\title{
Hepatic Artery Pseudoaneurysm Rupture with Acute Portal Vein Occlusion Treated with Coronary Stent Graft and Trans-splenic Portal Vein Stenting
}

\author{
Stephen K. Allison ${ }^{1}$ Arthie Jeyakumar ${ }^{2}$ \\ ${ }^{1}$ Department of Interventional Radiology, University of Washington \\ Medical Center, Seattle, Washington, United States \\ 2Department of Radiology, University of Washington Medical \\ Center, Seattle, Washington, United States
}

\begin{abstract}
Address for correspondence Stephen K. Allison, MD, Department of Interventional Radiology, University of Washington Medical Center, 1959 NE Pacific Street, Seattle, WA 98195, United States (e-mail: sallison@uw.edu).
\end{abstract}

\begin{abstract}
Keywords

- pancreaticoduodenectomy

- pseudoaneurysm

- bleeding

- stent graft

Delayed massive hemorrhage following pancreaticoduodenectomy is a potentially fatal condition. These patients are often not surgical candidates, and endovascular therapy is the primary option of treatment, usually trans-arterial embolization. However, the bleeding vessel can occasionally be treated with a stent graft rather than embolization. This decreases the risk of hepatic necrosis by preserving hepatic arterial blood flow, particularly in patients with compromised portal veins. The authors present a brief case report of delayed pseudoaneurysm rupture from a replaced proper hepatic artery after pancreaticoduodenectomy with resultant portal vein occlusion, caused by hematoma mass effect. The hepatic artery was treated with a coronary stent. During the same session, trans-splenic portal vein stenting was done.
\end{abstract}

\section{Introduction}

The most common complications after pancreatoduodenectomy are delayed gastric-emptying, pancreatic leakage, and intra-abdominal abscess formation. ${ }^{1}$ Hemorrhage following pancreaticoduodenectomy is less frequent; however, it is often a fatal complication. Hemorrhage can occur in the early or late postoperative phase. Hemorrhage within the first 24 hours after surgery is generally caused by a technical failure and needs immediate adequate hemostasis through a re-laparotomy. ${ }^{1}$ Hemorrhage in the late postoperative phase may be secondary to peptic ulceration, ulceration at an anastomosis, dehiscence of an anastomotic suture line, or an injured intra-abdominal vessel. Infection or enzymatic leakage from anastomotic failure may result in arterial wall erosions and predispose to pseudoaneurysm formation. The ligated gastroduodenal artery stump and the common hepatic artery are the most frequently involved vessels. Late onset of bleeding, also termed delayed massive hemorrhage, presents as sudden or intermittent severe intra-abdominal bleeding. ${ }^{1}$ Patients at this stage are often critically ill with postoperative and inflammatory adhesions making reoperation untenable. Endovascular arterial interventions include pseudoaneurysm embolization or exclusion via stent grafts. We present a case in which a coronary stent graft was used to emergently treat a ruptured replaced proper hepatic artery pseudoaneurysm in conjunction with trans-splenic portal vein stenting in the setting of post-pancreaticoduodenectomy hemorrhage with mass effect causing acute main portal vein occlusion.

\section{Case Report}

A 72-year-old man underwent pancreaticoduodenectomy complicated postoperatively by intra-abdominal abscess. He presented to the emergency room 7 weeks following surgery in hemorrhagic shock and acute liver failure. Pertinent laboratory values at presentation included a significant leukocytosis (46,000 white blood count $[\mathrm{WBC}] / \mu \mathrm{L}$ ), anemia (hematocrit 29\%), elevated lactate $(8 \mathrm{mmol} / \mathrm{L})$, and severe coagulopathy (international normalized ratio [INR] 5.1). His liver function revealed a total bilirubin of $3 \mathrm{mg} / \mathrm{dL}$, aspartate aminotransferase (AST) $4,920 \mathrm{U} / \mathrm{L}$, and alanine aminotransferase (ALT) $1,256 \mathrm{U} / \mathrm{L}$. Computed tomography (CT) demonstrated a contained pseudoaneurysm rupture arising from the proper received

September 4, 2018 accepted after revision

December 11, 2018

published online

March 18, 2019
DOI https://doi.org/

10.1055/s-0039-1681119

ISSN 2457-0214.
C2019 by Indian Society of Vascular and Interventional Radiology
License terms

() (1) $\ominus \circledast$ 
hepatic artery, occlusion of the main portal vein ( - Fig. $\mathbf{1}$ ), and multifocal hepatic infarcts. Angiography demonstrated a large bilobed pseudoaneurysm arising from a replaced proper hepatic artery, arising off the superior mesenteric artery, without visualization of intrahepatic vasculature ( - Fig. 2A). A microcatheter was navigated past the arterial defect, and injection confirmed a patent distal proper hepatic artery. A $4 \times 26-\mathrm{mm}$ GraftMaster RX coronary stent "JOSTENT" (Abbott Vascular) was deployed across the defect. Repeat angiography demonstrated pseudoaneurysm exclusion with preserved arterial perfusion to the liver (-Fig. 2B). Subsequent trans-splenic portography demonstrated occlusion of the main portal vein ( - Fig. $\mathbf{3 A}$ ). The occluded segment was traversed, and two overlapping Epic $12 \times 40-\mathrm{mm}$ self-expandable stents (Boston Scientific) were deployed. Repeat portography showed brisk flow into the portal system ( $\mathbf{- F i g}$. 3B). The trans-splenic access track was embolized with an 8-mm Nester coil (Cook Medical LLC).

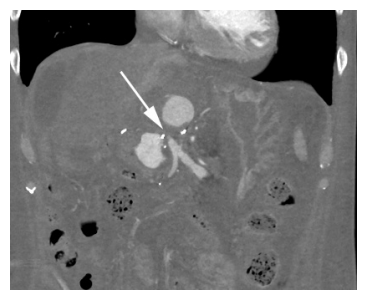

Fig. 1 Coronal CT image of main portal vein occlusion (arrow) at site of large bilobed pseudoaneurysm.
The patient was discharged home after a prolonged intensive care unit (ICU) stay for pneumonia. Hepatic artery stent graft patency and portal stent patency were confirmed by contrast-enhanced CT 205 days after placement. Unfortunately, because of progressive tumor recurrence, the patient was placed in hospice and died soon thereafter.

\section{Discussion}

Delayed massive hemorrhage following pancreaticoduodenectomy carries a high mortality rate. ${ }^{1}$ Several approaches to treat postoperative hemorrhage from pseudoaneurysms exist, including endovascular therapy, open repair, and conservative management. Lower mortality rates and decreased time in the intensive care unit have been reported in patients who undergo transarterial embolization and/or stent graft placement as compared with surgical repair. ${ }^{2}$ Factors considered in treatment planning include the location, size and shape of the aneurysm, tortuosity and diameter of the involved vessels, and presence of collateral flow to involved organs. Patients with pancreaticoduodenectomy are noteworthy in that they no longer have a gastroduodenal artery to provide collateral perfusion following embolization because the artery is sacrificed at resection. Therapeutic embolization may stop exsanguinating bleeding but may also carry the disadvantage of excluding the distal circulation. This may cause liver ischemia and even fatal hepatic necrosis, particularly in
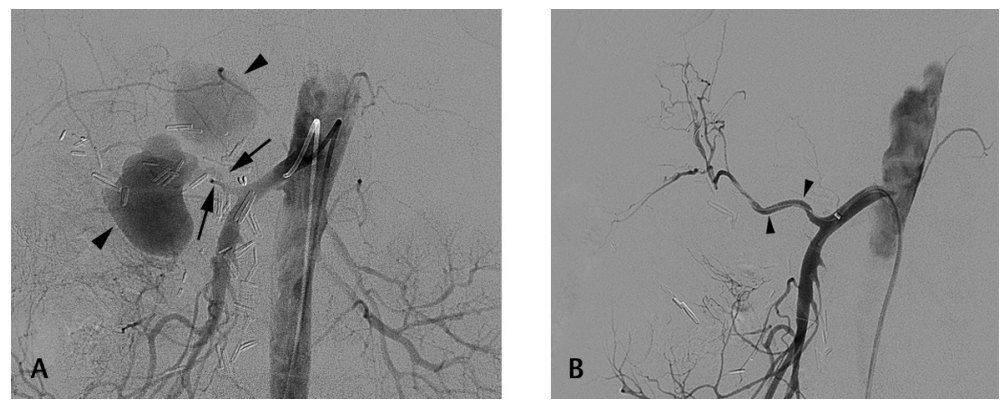

Fig. 2 (A) Digital subtraction angiography (DSA) demonstrating common hepatic artery trunk (arrows) and collections from hepatic artery rupture (arrowheads). (B) DSA demonstrating fully deployed GraftMaster RX (arrowheads) with newly patent hepatic artery without extravasation.
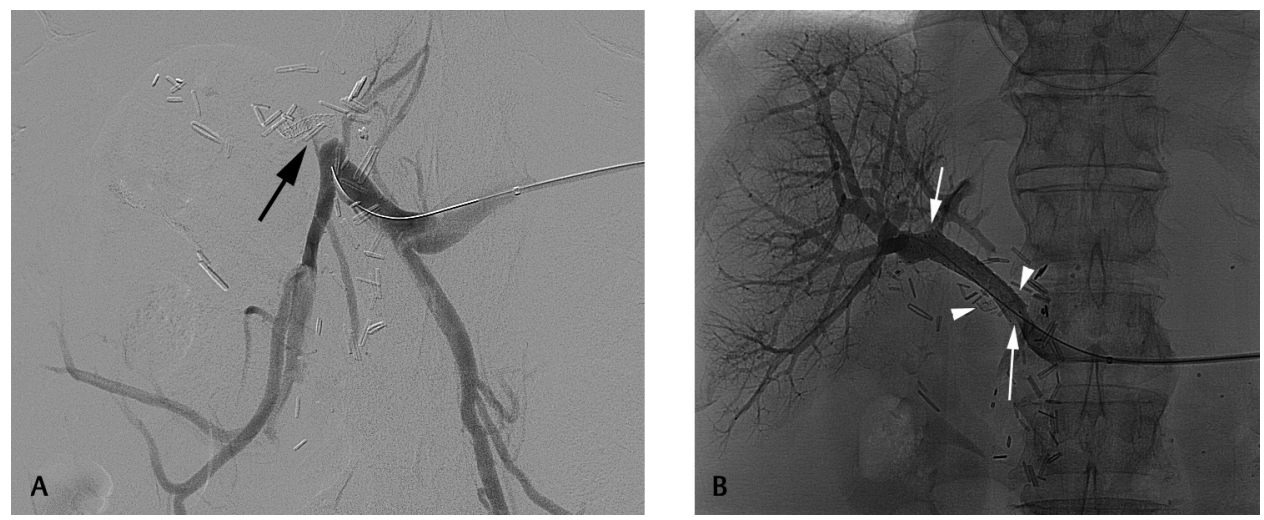

Fig. 3 (A) Digital subtraction venography demonstrating portal vein occlusion at site of rupture (large arrow points to deployed stent graft). (B) Portography demonstrating patent portal vein stents (arrows) with relation to common hepatic artery stent graft (arrowheads). 
patients with portal vein stenosis or thrombosis. ${ }^{3}$ This was a factor in treatment planning for our patient. For patients in whom endovascular management is unsuccessful, a temporary balloon occlusion of the involved artery can serve as a lifesaving method until transfer to the operating room. Risk factors associated with poor outcome after endovascular therapy include preprocedural coagulopathy, portal vein stenosis, and absence of visualized flow through hepatic arteries. ${ }^{4}$

The use of coronary stent grafts to exclude pseudoaneurysms is not frequently reported in the United States likely in part due to institutional review board (IRB) approval for use. Briguori et al demonstrated PTFE-covered coronary stents significantly reduce the necessity for emergency surgery compared with non-PTFE-covered stents for coronary perforations. ${ }^{5}$ Muraoka and colleagues reported a case in which a coronary stent graft was used to exclude a bleeding hepatic artery pseudoaneurysm at the anastomotic site following liver transplantation. While the procedure was technically successful, the patient died from sepsis 8 days later. ${ }^{6}$ Asai et al described treatment with a Jostent GraftMaster stent for delayed hemorrhage following pancreaticoduodenectomy for a common hepatic artery pseudoaneurysm on postoperative day 19 with stent patency seen at 9 months on $\mathrm{CT}^{3}{ }^{3}$ The largest series was published by Wang et al, who reported their experience in nine patients with delayed massive hemorrhage from ruptured hepatic artery pseudoaneurysm after pancreaticoduodenectomy, using the same stent graft (Jostent, GraftMaster) for emergency vessel repair retaining patency of hepatic artery. Instead of coil embolization, stent graft was placed to exclude hepatic pseudoaneurysm because all patients had portal venous obstruction. ${ }^{7}$ Six of nine patients survived with patent stents during a mean follow-up time of 10.5 months (range: 4-16 months). Our case is the only one to report stent graft placement in conjunction with reestablishing in-line portal flow.

While many stent grafts exist, the size of the vessels involved in the pancreatic bed may prevent use of the stent grafts that are designed for use in the periphery; iCAST (Atrium Medical Corporation), Viabahn, and Viabahn VBX (W. L. Gore \& Associates, Inc), all of which have 5-mm-diameter stents as their smallest size. Furthermore, Viabahn is a self-expandable device on a 0.018 -in platform with the shortest available length of $5 \mathrm{~cm}$. Although the balloon-expandable devices have shorter stent lengths, they are on larger, less flexible 0.035 -in platforms that may not allow for delivery of the stent graft in some visceral locations. In this case report, a PTFE-covered coronary stent was chosen to treat the proper hepatic pseudoaneurysm to preserve hepatic arterial flow given the patient's portal vein occlusion. Therapeutic embolization would not have been ideal in this instance because of the risk of hepatic necrosis. ${ }^{1}$ Jostent/GraftMaster is a covered stent, ranging 2.8 to $4.8 \mathrm{~mm}$ in diameter and 16 to $26 \mathrm{~mm}$ in length. This stent was chosen because of its small diameter and short length, which was necessary for placement within the proper hepatic artery. Flexibility of the 0.014 -in platform also allowed for delivery to an arterial segment after significant tortuosity. After the life-threatening hemorrhage was controlled, portal flow was reestablished with stent placement across the main portal vein to also help prevent hepatic necrosis.

In conclusion, this report describes lifesaving placement of a coronary stent graft for treatment of postoperative hepatic artery pseudoaneurysm in conjunction with portal vein stenting with documented patency at 205 days. GraftMaster $\mathrm{RX}$ is authorized by U.S. federal law for use in the treatment of free perforations, defined as free contrast extravasation into the pericardium, in native coronary vessels, or saphenous vein bypass grafts $\geq 2.75 \mathrm{~mm}$ in diameter. However, this stent is approved by the Food and Drug Administration (FDA) with a humanitarian device exemption and requires IRB auspices for use. Given our case and the other existing reports, its potential use in noncardiac life-threatening situations should be known.

\section{Conflict of Interest \\ None.}

\section{References}

1 de Castro SM, Kuhlmann KF, Busch OR, et al. Delayed massive hemorrhage after pancreatic and biliary surgery: embolization or surgery? Ann Surg 2005;241(1):85-91

2 Ding X, Zhu J, Zhu M, et al. Therapeutic management of hemorrhage from visceral artery pseudoaneurysms after pancreatic surgery. J Gastrointest Surg 2011;15(8):1417-1425

3 Asai K, Watanabe M, Kusachi S, et al. Successful treatment of a common hepatic artery pseudoaneurysm using a coronary covered stent following pancreatoduodenectomy: report of a case. Surg Today 2014;44(1):160-165

4 Hasegawa T, Ota H, Matsuura T, et al. Endovascular treatment of hepatic artery pseudoaneurysm after pancreaticoduodenectomy: risk factors associated with mortality and complications. J Vasc Interv Radiol 2017;28(1):50-59.e5

5 Briguori C, Nishida T, Anzuini A, Di Mario C, Grube E, Colombo A. Emergency polytetrafluoroethylene-covered stent implantation to treat coronary ruptures. Circulation 2000;102(25):3028-3031

6 Muraoka N, Uematsu H, Kinoshita K, et al. Covered coronary stent graft in the treatment of hepatic artery pseudoaneurysm after liver transplantation. J Vasc Interv Radiol 2005;16 (2 Pt 1):300-302

7 Wang MQ Liu FY, Duan F, Wang ZJ, Song P, Fan QS. Stentgrafts placement for treatment of massive hemorrhage from ruptured hepatic artery after pancreaticoduodenectomy. World J Gastroenterol 2010;16(29):3716-3722 